\title{
XAVIER ZUBIRI Y MARÍA ZAMBRANO: \\ DE LA CRISIS EUROPEA A UNA REFORMA DEL ENTENDIMIENTO ${ }^{1}$
}

\author{
PAMELA SOTO GARCÍA Y RICARDO ESPINOZA LOLAS \\ Pontificia Universidad Católica de Valparaíso
}

\begin{abstract}
RESUMEN: La articulación y cruce de vida y obra, de María Zambrano y Xavier Zubiri, en tanto representantes de la primera etapa de la Escuela de Madrid, permiten en este artículo abordar la originalidad del pensamiento español desde un horizonte antropológico, a través de la crítica que ambos filósofos realizan al yo cartesiano. Esta perspectiva de análisis tiene como eje de referencia la crisis europea, que constata Ortega y Gasset, como un elemento que da cuenta de toda una época, y que tiene como uno de sus principales representantes al sujeto solipcista de la modernidad.
\end{abstract}

PALABRAS CLAVE: Zambrano, Zubiri, Ortega y Gasset, crisis europea, yo

\section{Xavier Zubiri and María Zambrano: from the European Crisis to a reform of enlightenment}

ABSTRACT: Life and work of Maria Zambrano and Xavier Zubiri as representatives of the first period of «Escuela de Madrid», crosses and articulates in this paper research, through the criticism that both philosophers do about Cartesian self, allowing thus to address the originality of Spanish thought from an anthropological perspective. The solipsism, which is verified by Ortega y Gasset, is an evidence of the European crisis that outstands in modernity and the point of view of this analysis.

KEY WORDS: Zambrano, Zubiri, Ortega y Gasset, European crisis, Cartesian self

La Escuela de Madrid durante la primera mitad del siglo xx se estableció como un horizonte de renovación para el pensamiento español ${ }^{2}$. Este horizonte propone la instauración de nuevos referentes filosóficos que permitieran el desarrollo de una reflexión situada y propia desde la lengua y el territorio español, lo que en una primera época permitió que se identificara esta búsqueda con la metáfora del «logos del Manzanares $»^{3}$. Si bien esta Escuela se organiza a partir del pensamiento de

1 Este artículo es parte del Proyecto Fondecyt Nº: 1140973: «Realidad y técnica en Zubiri».

2 «Ortega se dio de lleno a la reforma de la Facultad de Filosofía y Letras de Madrid, en unión con otras figuras eminentes del Profesorado, tal como Américo Castro. La reforma de los estudios filosóficos fue llevada a cabo, junto con Ortega, por profesores que había hecho que la enseñanza - la vida- de la filosofía fuese plena realidad, que fuese innecesario ir a otro país a estudiar filosofía en serio». Zambrano, M. (2004) «La razón que se busca (A propósito de la razón vital)», Revista de Occidente, 276, p. 118.

3 «Hay también un logos del Manzanares: esta humildísima ribera, esta líquida ironía que lame los cimientos de nuestra urbe, lleva, sin duda entre sus pocas gotas de agua alguna gota de espiritualidad». ORTEga y GaSSET, J. (1983), «Meditaciones del Quijote» en Obras Completas, I, Madrid: Alianza, p. 322. En todo caso la expresión no solamente es bellísima, y es un gran acierto de Ortega, sino que además muestra la «ambición» del proyecto filosóficopolítico que desde Madrid, y su río, así como en otras grandes civilizaciones, se quiere expandir no solamente al resto de Europa sino a toda Latinoamérica (y el Mundo). Y por eso 
José Ortega y Gasset, ésta no culmina en la reflexión del filósofo sino que impacta en toda una generación de pensadores ${ }^{4}$, que a partir del nuevo contexto que ofrece el pensamiento español comienzan a desarrollar sus propias filosofías, entre estos pensadores se encuentran: Xavier Zubiri y María Zambrano.

Revisar el cruce entre ambos filósofos, desde lo biográfico y teórico, permitirá ver el impacto de esta Escuela para el desarrollo del pensamiento español y latinoamericano a lo largo del siglo xx, además de realizar una revisión de algunos de los tópicos por los que ambos filósofos transitaron en conjunto y que permitieron el desarrollo posterior de sus pensamientos, situando esta reflexión en el problema de la «persona».

\section{Algunos antecedentes acerca de la Escuela de Madrid}

Julián Marías publica a fines de los años cincuenta un libro titulado La escuela de Madrid ${ }^{5}$. Con este nombre pretende dar cuenta de un hecho radical acontecido en la vida filosófica española a comienzos del siglo xx, debido a que esta Escuela a la que alude el pensador español, se caracteriza por la búsqueda de una enseñanza filosófica que actualice y sitúe a esta reflexión disciplinar desde un nuevo lugar. «La transformación del país es concebida por el joven Ortega como el proceso mediante el cual España se incorpora a la cultura europea. Así queda marcada la que él considera su vocación pública como intelectual, su destino educador, casi de reformador social: empeñarse en poner a España a la altura de la cultura de Europa» (Sánchez Escámez, 1993, p. 808)

este proyecto va de la mano no solamente desde la Universidad Central de Madrid, sino que está acompañado de muchos jóvenes y brillantes investigadores. Además de saber lenguas europeas modernas (Ortega y Zubiri, por ejemplo, dominaban el alemán; lengua fundamental para el desarrollo filosófico del siglo xx) y clásicas (griego y latín); el equipo se dedicaba a traducir libros fundamentales no solamente de la filosofía sino de las ciencias. Y con eso se esperaba que España diera un salto cualitativo y saliera de la oscuridad en la que se encontraba. Y el proyecto también se expresaba en la ya mítica Revista de Occidente (fundada en 1923); la cual sigue en la actualidad vigente. Una revista que en su nombre también indicaba esa "ambición» de abrir el pensamiento y de construir pensamiento efectivo, así como lo estaban haciendo los ingleses, los franceses y los alemanes de la primera mitad del siglo xx.

4 «[Ortega] En el ámbito académico es la personalidad más influyente de la filosofía española de su tiempo. En torno a él, bajo la influencia de su filosofía y personalidad, se constituye la llamada "Escuela de Madrid". Manuel García Morente, Xavier Zubiri y José Gaos son con Ortega los titulares de las cátedras de filosofía de la Universidad madrileña. Cualquier conocedor de la cultura española sabe la importancia de esos nombres. Si a ellos añadimos lo de Luis Recanséns, María Zambrano, Joaquín Xirau y Julián Marías, que por uno u otro motivo están en relación con la Escuela». Escamés SÁncheZ, J.J. (1993) «Ortega y Gasset (1883-1955)», Perspectivas: revista trimestral de educación comparada, XXII. París: UNESCO Oficina internacional de Educación, p. 818.

5 Este texto fue publicado por Marías el año 1959, y en él además de sendas reseñas acerca del influjo de Ortega y Zubiri en el pensamiento español del siglo xx, presenta la ampliación de otros textos publicados en la obra de 1955 Filosofía actual y existencialismo en España. 
Esta escuela se organiza a mediados de 1915 a partir de la figura de José Ortega y Gasset. No obstante, lo que Marías señala en su texto es que la denominación «Escuela de Madrid» no alude a un tipo de orteguismo, sino más bien designa a un grupo de intelectuales que participaron en la renovación del pensamiento filosófico español, sin embargo, es necesario tener presente que esta renovación surge a partir del influjo y ímpetu de trabajo propuesto por Ortega en las diversas acciones que emprendió para ejecutar este proceso de cambios en el pensamiento español.

Esta renovación que impulsaba Ortega tiene diversas vertientes. Desde lo político, por ejemplo, surge a partir de la preocupación por repensar a España, desde su situación de decadencia imperial, a partir de la pérdida de Cuba y Filipinas a finales del siglo XIX. Sin embargo, a diferencia de la generación del 986, quienes también daban cuenta de esta panorama, Ortega considera que es posible revertir esta decadencia en la que se encuentra sumida España, a partir de una apuesta intelectual de gran alcance, pues en ésta no sólo incluirá a España, sino también a las tierras americanas. Y tal apuesta, incluso, quiere ser parte fundamental de Europa.

Desde esta perspectiva la Escuela de Madrid era un proyecto no solo filosófico sino de desarrollo del pensamiento de toda España (de Europa) y con ello de la misma Latinoamérica ${ }^{7}$. Pues era un proyecto en donde se tenía que colo-

6 En 1913 Azorín acuña el término Generación del 98 para hacer referencia a un grupo de intelectuales que adoptaron una actitud crítica ante la situación política y social española originada desde fines del siglo XIX, producto de la finalización del período del imperialismo colonial, tras la pérdida de Filipinas, Puerto Rico y Cuba en 1898, fecha a partir de la cual Azorín nomina a esta generación. Entre los principales exponentes de esta generación se encuentran: Pío Baroja, Azorín (José Martínez Ruiz), Ramiro de Maetzu, Ángel Gavinet, Miguel de Unamuno y Antonio Machado, entre otros. En 1901 difunden Baroja, Arozín y Maetzu un Manifiesto, en la que denuncian la descomposición de su época y en especial la de España, que es afrontada entre una crítica al fracaso y una exaltación de su pueblo y paisaje, además de dar primacía a temas existenciales. Zambrano, en el texto Pensamiento y poesía en la vida española, publicado por primera vez en septiembre de 1939, declara: «Durante más de dos siglos, España se va desintegrando, debilitando con un ritmo creciente que la hace desembocar en el siglo XIX reducida a un estado en que viene a ser problema su existencia misma». ZAMBRANo, M. (2004), Pensamiento y poesía en la vida española. Madrid: Biblioteca Nueva, p. 221.

7 «El perspectivismo cultural orteguiano hacia América se inició abriendo un horizonte historicista con estratos históricos específicos. Podríamos dividir el proceso en cinco direcciones: la primera enfocaba la realidad social del aluvión migratorio. La segunda viraba hacia las Atlántidas pueblos antiguos sumergidos y evaporados en pasados ancestrales [...]. La tercera pasará por las transformaciones del hombre masa en América, que le conducen a un fino análisis de una nueva casta burocrática en Argentina, y a la uniformidad del hombre standard norteamericano, que se perfila con más precisión en su Rebelión de las masas. El cuarto eslabón, que procede de los anteriores estratos, le conducirá hacia el tipo del pionero americano, el que vivió y condujo la vida colonial anterior al fenómeno migratorio y que también poco se asocia al conquistador o el administrador de las colonias. Una quinta categoría [...] será el modelo imperialista de estas emergentes repúblicas a las cuales vincula al fenómeno del Imperio romano, emporio universal de civilización aglutinadora de razas con ambición de convertirse en potencia internacional». CAMPOMAR, M. (2004) «El perspectivismo americano de Ortega y Gasset», Revista de Occidente, 276, pp. 157 y 158. 
car a España en la vanguardia misma de Europa; ser como Francia y para ello tanto la filosofía como las ciencias en general eran fundamentales. Se veía a España literalmente atrasada en el continente. Desde esta perspectiva, la creación por parte de Ortega de la Revista de Occidente, cumplía con varias de estas funciones; una de ellas era poder traducir en formato de libros grandes obras filosóficas y científicas que no era posible encontrarlas en español, lo cual era un escándalo ${ }^{8}$. Para Ortega, España no saldría de su oscuridad mientras no se leyera ni estudiara la producción científico-filosófica europea ${ }^{9}$. Gracias a esta iniciativa autores como Einstein y Husserl (entre muchos otros) ${ }^{10}$ pudieron por fin ser leídos en España y toda Latinoamérica (como se sabe eran muy pocos en esos años los que podían leer directamente alemán; Ortega era uno de esos privilegiados $)^{11}$.

8 «El Espectador es la revista que hace en soledad. España junto a Manuel Azaña y []. Años después la Revista de Occidente, con su editorial de selección, de "vanguardia". Inspira y orienta la Editorial Espasa Calpe, que hacía asequibles los clásicos universales y las últimas avanzadas del pensamiento». Zambrano, M. (2004), «La razón que se busca», Revista de Occidente, 276, p. 107. Entre los libros publicados por la editorial de la Revista de Occidente durante esta época se encuentran: H. Keyserling (1926), El mundo que nace; G. Simmel, (1926-27), Sociología: estudios sobre las formas de socialización; F. BRENTANo (1927), El origen del conocimiento moral; C. G. Jung (1927), Lo inconsciente: en la vida psíquica normal y patológica; A. Messer (1927), De Kant a Hegel; W. FranK(1929), Redescubrimiento de América; E. HusserL (1929), Investigaciones lógicas; H. HöFFding (1930), Sören Kierkegaard; B. Russell (1931), El panorama científico; J.G Fichte (1934), Los caracteres de la edad contemporánea; M. SCHELER (1934), Muerte y supervivencia; F. BRentano (1936), El porvenir de la filosofía; entre otros.

9 El impacto de la Revista de Occidente no sólo se apreció en España, sino también en América Latina y Zubiri participó en ella como colaborador y traductor de Suárez, Descartes, Pascal, Hegel, Brentano, Scheler, Messer, March, Schördinger y De Broglie. «Para América Latina la Revista de Occidente significó un ponerse a la altura de los tiempos. De pronto se encontraba a su inmediato alcance lo mejor de la reacción cultural europea y en español». Modin, I. (1994), Ortega y Gasset y la cultura latinoamericana. México: FCE, p. 33.

10 La Revista de Occidente es fundada por José Ortega y Gasset en julio de 1923 y él mismo la dirige hasta el estallido de la Guerra Civil Española en julio de 1936. Durante este primer período de la Revista contempló la publicación de 157 números. Cfr: J. P. Camazón LinACERo (2000), «La crisis europea en Revista de Occidente (1923-1936)», Espacio, Tiempo y Forma, 13, pp. 369-391.

11 Si España estaba en la oscuridad, el resto de Latinoamérica estaba completamente atrasada respecto al gran desarrollo de la propia concepción de la sociedad civil, pues los saberes fundamentales que desarrollan la cultura no estaban aconteciendo. No se sabía casi nada de las ciencias (relatividad, cuántica, teoría de conjuntos, teoría numérica, biología, etc.), tecnologías (éstas se expresaban desde el desarrollo de armamentos a fármacos pasando por todo el despliegue agropecuario) y en especial de las filosofías (idealismo, criticismo, positivismo, pragmatismo, marxismo, psicoanálisis, vitalismo, psicologismo, analítica, fenomenología, hermenéutica, etc.). Latinoamérica no estaba en la oscuridad, estaba en un hoyo profundo a miles de km del despliegue cultural europeo. Y el proyecto de Ortega con su «logos del Manzanares» era realmente un salto cualitativo en el orden del conocimiento (y esto mucho antes de las nuevas tecnologías y de la era digital en la que estamos); salto que en pocos años podía ejercer un gran impacto de «iluminar» ese hoyo en el que se vivía como si fuera lo más normal. La era de la superstición, el fanatismo y la arbitrariedad daba paso a una sociedad más libre y ciudadana. 
También la Revista de Occidente permitió la producción de pensamiento escrito en español; son memorables, por ejemplo, los artículos que Zubiri escribió en dicha revista ${ }^{12}$. Ortega sabía por la Universidad, como por las charlas y tertulias, que a través de la publicación de la Revista de Occidente se estaba produciendo algo nuevo en España que podría permitir insertarla en Europa desde cierto horizonte de pensamiento común (entre hermenéutica, fenomenología y ciencias). Julián Marías describe la situación previa a esta organización de la Escuela de Madrid del siguiente modo:

Por entonces, la filosofía en España no existía. Epígonos mediocres de la escolástica, residuos informes de positivismo, místicas tinieblas del krausismo, habían desviado el pensamiento español de la trayectoria viva. España permanecía, por decirlo así, al margen del movimiento filosófico (Marías, 1959, p. 12).

Este contexto permite dar cabida a la renovación del pensamiento español que Ortega tanto anhelaba y que a través de intelectuales como Xavier Zubiri y María Zambrano (entre otros) emprenden con fuerza una reforma filosófica que se comienza a fraguar desde las aulas de la Universidad Central de Madrid. Esta renovación tiene como fecha de inicio el regreso de Ortega a Madrid en $1911^{13}$, período a partir del cual el filósofo se ocupa de renovar e implantar una

12 Entre la nómina de colaboradores durante la primera etapa de la Revista de Occidente es posible destacar: al físico Albert Einstein, al médico psiquiatra y psicólogo Carl Gustav Jung, a los economistas Werner Sombart y Luwdin von Missen, a los filósofos Max Scheler, Xavier Zubiri, José Gaos, Manuel García Morente, Carl Schmitt, al poeta Pablo Neruda, a los escritores José Luis Borges, Paul Valery y Waldo Frank, a un importante número de los integrantes de la generación del 98 como Azorín o Baroja; y a toda la generación del 27. Cfr: Camazón Linacero, J.P. (2000), "La crisis europea en Revista de Occidente (1923-1936)», Espacio, Tiempo y Forma, 13, pp. 369-391. Entre las reseñas, traducciones y artículos de Zubiri de esta época editados por la Revista de Occidente y Editorial se encuentran: Reseña «P. L. Landsberg: La Edad Media y nosotros» de 1925; Reseña «F. Brentano: Psicología» de 1926; Traducción de A. Messer «Filosofía Antigua y Medieval» de 1927; Artículo «Sobre el problema de la filosofía» de 1933; Traducción y Prólogo A. March "La física del átomo. Iniciación en las nuevas teorías»; Artículo «En torno al problema de Dios» de 1935; Traducción M. Scheler «Muerte y Supervivencia, Ordo amoris» de 1935; Prólogo y Traducción de G.W.F. Hegel «Fenomenología del espíritu»: Advertencia Preliminar a F. Suarez "Disputaciones metafísicas sobre el concepto de ente» de 1935; Prólogo y Traducción de F. Brentano "El porvenir de la filosofía» de 1936. En el caso de Zambrano lo que publica en la Revista de Occidente durante este período es: «Lou Andreas Salomé: Nietzsche» de 1933; «Por qué se escribe» de 1934.

13 Ortega en 1911 obtiene la cátedra de Metafísica de la Universidad de Madrid, única en España, previo a este período se encontraba en Alemania realizando estudios de especialización en filosofía. Ortega en relación a su estancia universitaria comenta: «Yo había estudiado un semestre en Leipzig [...] Al semestre siguiente fui a Berlín. Vivía con una pequeña pensión del Estado español que había obtenido mediante un concurso [...] Hacia 1908 estuve un año entero en Marburg y en 1911 volví [...]. Marburg era el burgo del neokantismo. Se vivía dentro de la filosofía kantiana como en una ciudadela sitiada [...] El gobernador de la ciudadela, Cohen, era una mente poderosísima. La filosofía alemana y todo el mundo tiene una gran deuda con él [...] Cohen obligó a tomar contacto íntimo con la filosofía difícil y, sobre todo, renovó la voluntad de sistema, que es lo específico de la inspiración filosófica [...] Sin 
nueva mirada sobre el quehacer filosófico español, y junto a sus discípulos y colegas de trabajo, hacer de la Universidad Central de Madrid un lugar donde se cultiva la filosofía con intensidad. Estos rasgos están presentes en la obra de ambos pensadores, quienes tempranamente comienzan a elaborar desde este horizonte de búsqueda un pensamiento propio.

Es necesario destacar que antes de esta renovación de la reflexión filosófica española, a mediados del siglo XIX, España había incursionado en una reforma del pensamiento a partir de la introducción del krausismo ${ }^{14}$, transformación que fue difundida por la Institución Libre de Enseñanza e impulsada por Sanz del Río ${ }^{15}$. El krausismo español influyó radicalmente en aquello que Ortega y Gasset denominará el «ser español», pero es necesario precisar que esta doctrina adquiere ribetes particulares en España y que no caracteriza, propiamente, el pensamiento de Krause, ya que, el krausismo español se define más bien como el contrapunto al panorama filosófico dominante en España del siglo XIX, la escolástica y el neocatolicismo; por ende, aquello que se intenta realizar a partir de la implementación de esta doctrina es generar una fundamentación humanista para la vida práctica, como el derecho o la pedagogía, pero no se persigue con la introducción de este pensamiento la implementación de un desarrollo filosófico.

embargo, tengo que añadir tres cosas. La primera es que, propiamente hablando, en Marburg no se enseñaba filosofía. Era preciso saberla ya de antemano [...].La segunda cosa es que, salvo aquella fertilísima voluntad de sistema, los neokantianos no disparaban las mentes jóvenes hacia problemas abiertos que fuese posible o interesante pensar [...] La tercera cosa... la tercera cosa es una cosa que no me atrevo a decir ahora. Es más grave». ORTEGA y Gasset, J. (1983), «Prologo para alemanes», en Obras Completas, VIII. Madrid: Alianza, pp. 26-27.

14 María Zambrano en «España despierta soñándose», capítulo de su libro autobiográfico Delirio y Destino expone con detalle cómo se instala el krausismos en España. Nos parece de suma relevancia traer esta descripción que realiza la autora, pues ésta nos situará en el horizonte histórico previo a la organización de la Escuela de Madrid, lo que permite entender por qué surge una figura como Ortega y Gasset, quien fue educado a partir de esta nueva mentalidad española que se impone por medio del krausismo: «Tal debió ser el verdadero sentir que a un Sanz del Río le hizo ir por el año cuarenta del siglo pasado a Alemania en busca de una filosofía. Y trajo una filosofía secundaria, el krausismo, de tan difícil expresión que llegaba hasta lo ininteligible al ser traducida al castellano [...] España como detenida que quedó en el siglo XvII, no ha sido nunca un país de burguesía; la "clase media» es otra cosa. Y así nos ha faltado fuerza creadora en la ciencia, en la industria, en los modos de vivir que alcanzaron auge en el siglo XIX de Europa, en Francia, sobre todo, en Inglaterra, en la misma Alemania, aunque de matiz un tanto diferente. Lo sabíamos, lo supusieron aquellos hombres que quisieron con el krausismo como instrumento moral elevar el nivel de la vida española, crear esta clase nueva o casi nueva: profesores, científicos, intelectuales, artistas, que formaban parte de la sociedad viviente y que encontraron en ella su acicate y, en el Estado, su sostén». Zambrano, M. (1989), Delirio y Destino. Madrid: Mondadori, pp. 75 y ss.

15 Julián Sanz del Río, filósofo y jurista español (1814-1869), que a mediados del siglo XIX introdujo en España la filosofía de Krause como contrapartida al dogma católico imperante, cuya repercusión no sólo influyó en toda España a través de la Institución Libre de Enseñanza, sino también influyó en América Latina, en especial en Puerto Rico, a través de la figura de Eugenio María de Hostos. 
Luego, de esta primera transformación del pensamiento español que se impone a través del krausismo, adviene una segunda modificación realizada desde la Facultad de Filosofía y Letras de Universidad Central de Madrid, que esta vez sí persigue la generación de pensamiento filosófico, debido a que el ejercicio que se realiza consiste en la construcción de un pensamiento forjado en España, cuya principal riqueza radica en la originalidad de sus integrantes, pues no existe un cuerpo doctrinal que se pretenda implementar a través de esta Escuela, debido a que la obra de sus principales exponentes no puede ser homologada en un único sistema de pensamiento, ni reducida a la localidad de su ejercicio, sino que el trabajo que se realiza en sus aulas, responde a la crítica y deconstrucción de ciertos referentes filosóficos, que le permite al grupo de filósofos que participa en ella elaborar sus pensamientos. En este texto en particular se hará referencia a la crítica que realizan estos pensadores al «yo» de la modernidad, y que los conduce a repensar una modificación del carácter de lo humano, primero desde la esfera de una reforma del entendimiento, para luego en una segunda etapa presentar a la «persona» como garante de la salida del pensamiento, sustancialista, dualista e, incluso, fenomenológico acerca de lo humano (o sea, ni Aristóteles, ni Descartes, ni Husserl son suficientes), inaugurando con ello el carácter físico de la persona (como diría Zubiri).

\section{XaVier Zubiri y María Zambrano ENTRE unA RElación BIOGRÁFICA y FILOSÓFicA}

María Zambrano, durante su formación en la Escuela de Madrid recibe instrucción por parte de reconocidos docentes: Ortega y Gasset, Cossío ${ }^{16}$, Besteiro $^{17}$, García Morente ${ }^{18}$, Zubiri, Cejador ${ }^{19}$. Sin embargo, es la propia filósofa quien se encarga de señalar en Cartas a la Pièce. Correspondencia con Agustín Andreu a Ortega y Zubiri como sus maestros. En esta carta describe, brevemente, el tipo de relación que sostuvo con ambos: «Los dos maestros. Quizá tenga que rectificar lo que a dos discípulos míos dije delante de Bergamín, con cierta

16 Manuel Bartolomé Cossío (1858-1935). Continuador del movimiento krausista e historiador del arte. En 1908 publicó El Greco y en 1914 El Entierro del Conde de Orgaz.

17 Julián Besteiro catedrático y político español especialista en el área de Lógica. El año 1939, junto a otros académicos como Wenceslao Roses y José Gaos fue exonerado de su cargo universitario por el gobierno franquista.

18 Manuel García Morente (1888-1942) Profesor de ética en la Escuela de Madrid, tradujo a un buen número de obras filosóficas al español, entre las que destacan la obra de Spengler, Kant, Husserl, Brentano entre otros. En 1932 publica Ensayo sobre el progreso, y en 1935 Ensayo sobre la vida privada.

19 Julio Cejador (1864-1927) filólogo y crítico literario. En el año 1900 publicó Gramática Griega, en 1911 Introducción a la ciencia del lenguaje, y en 1905-1906 en dos tomos La lengua de Cervantes. Estos datos bibliográficos sobre María Zambrano corresponden al trabajo que realiza el estudioso y amigo personal de la filósofa Moreno SANZ, J. (2004), «Guías y Constelaciones» en María Zambrano 1904-1991. De la razón cívica a la razón poética. Madrid: Residencia de Estudiantes, pp. 209-251. 
esperanza de que se lo refiriera al superviviente [Zubiri] "Tuve dos maestros: el uno me veía, el otro no me podía ver. Y quizá: el uno no podía acabar de verme y el otro me vio un instante, un solo instante"» (Zambrano, 2002, p. 87).

Esta última frase hace alusión al breve e intenso tiempo que Zambrano y Zubiri compartieron en la Escuela de Madrid, debido a que al poco tiempo de iniciada esta amistad (que llamaba la atención de todos, Zubiri era un sacerdote y ella siempre tuvo un rasgo «revolucionario») el filósofo parte a Alemania, lo que no sólo los distancia físicamente, sino que a partir de ese viaje no volverán a retomar este vínculo afectivo.

En la biografía del filósofo, Xavier Zubiri. La soledad sonora, Jordi Corominas y Joan Albert Vicens, dedican varias páginas que dan cuenta de la relación entre ambos pensadores en la que destaca una temprana cercanía. «Zubiri se da cuenta de su ascendente sobre las mujeres [...] No hace demasiadas concesiones a las alumnas que le parecen poco valiosas, sin auténtico interés por el estudio y la cultura. [...] En cambio, trata con toda delicadeza a María Zambrano, en la que reconoce un auténtico espíritu intelectual». (Corominas \& Vincens, 2006, p. 188)

En Delirio y destino, autobiografía escrita en la Habana en 1951, la autora detalla pormenores sobre el trabajo filosófico que emprende con cada uno de sus maestros, aludiendo al modo como ambos realizan sus clases en la época.

«Trabajosamente había asistido sin perder apenas unas clases de Metafísica con Ortega de tan deslumbrante claridad... era tan claro y sin embargo apenas había entendido alguna cosa. Un curso, todo un curso sobre la «Crítica de la Razón Pura». Había asistido más angustiosamente aún al curso sobre la «Metafísica» de Aristóteles del joven maestro Xavier Zubiri». (Zambrano, 1989, p. 32)

María Zambrano conoce a Xavier Zubiri en la Facultad de Filosofía y Letras de la Universidad Central de Madrid, por esos años el joven pensador comenzaba a impartir la cátedra de "Historia de la Filosofía» ${ }^{20}$. A este vínculo entre profesor y alumna se agrega una relación externa que puede ser catalogada como de maestro y discípula. Zambrano en una carta a Agustín Andreu relata, detalladamente, su primer encuentro con el filósofo:

Te diré que Zubiri [...] Tenía el sentido del ser en grado sumo — del SERprofundizaba, exponía sin concesión pedagógica alguna. Le contesté a la pregunta que nos hizo a los cinco o seis auditores, «Explica Vd. Filosofía al modo matemático». Y le gustó mucho. Comenzó llanamente así su profesorazgo. Llegué tarde a ésta su $1^{\text {a }}$ clase y se levantó para buscarse (sic) y ofrecerme una silla. Poco después nos dijo a mi compañera y a mí que fuéramos a una cierta hora a la Secretaría de la Facultad para exponerles nuestras dudas — solo a las dos- y le gustó más cuando le repliqué —claro que suavemente- a su

20 Zubiri durante 1927 imparte los cursos de «Historia de la Filosofía Griega», «El platonismo del Renacimiento» y un seminario monográfico sobre Descartes. Zambrano también realiza con Zubiri un curso dedicado a la Metafísica de Aristóteles. Estos datos han sido extraídos de aquello que la autora declara en sus libros Delirio y Destino y El hombre y lo divino. 
«Vamos a ver: expónganme sistemáticamente sus dudas»: «Si fueran sistemáticas, no serían ya apenas dudas». Y creo que esto fue lo que le llevó a proponerme que hiciera con él la Tesis, si otra idea yo no tenía. Tardó, tuve la impresión que a propósito, en darme un papelito que decía «La teoría de la definición en Aristóteles y en Hegel» y no me inmuté21. (Zambrano, 2002, pp. 87 y 88$)$.

El trato entre Zubiri y Zambrano no termina sólo en las aulas de la Universidad, debido a que prontamente comienzan a reunirse fuera de este recinto como un método que utiliza Zubiri para a apoyar a Zambrano con sus estudios de filosofía, luego que la pensadora le confidenciara estar pasando por una crisis personal que le ha hecho repensar su permanencia en la Facultad. Estas reuniones extra universitarias se mantendrán por más de un año, y marcarán para siempre el decurso del pensamiento de la filósofa, lo que se ve expresado en el influjo que la obra de Baruj Spinoza tiene en su pensamiento, y que la lleva a plantear una tesis doctoral que nunca terminó acerca de la salvación del individuo en Spinoza $^{22}$, debido a que en este periodo de encuentros fuera de la universidad, se dedican a leer con detalle la ética de Spinoza, y la sexta Eneada de Plotino ${ }^{23}$.

21 En la biografía de Xavier Zubiri Xavier Zubiri: La soledad sonora se relata el primer encuentro entre ambos pensadores, así como también su posterior relación de trabajo, sin embargo, entre ambos escritos existe una diferencia en relación con el tema de tesis propuesto por Zubiri a Zambrano, ya que, en la obra sobre la vida de Zubiri se señala que el tema es «La teoría de la definición en Aristóteles y Santo Tomás», en cambio, la carta que dirige Zambrano a Andreu indica que la propuesta consistía en desarrollar una investigación sobre: «La teoría de la definición en Aristóteles y Hegel». Existe una nota en el libro El hombre y lo divino que puede ser aclaratoria en este sentido, pues en ella se da cuenta de la relación que establecía Zubiri entre Aristóteles y Hegel: «La analogía entre Aristóteles y Hegel, en cada uno de sus respectivos horizontes, es una idea que escuché insistentemente hace años a mi maestro de historia de la filosofía, Xavier Zubiri», lo que conduce a pensar que Zubiri tenía este problema ya instalado al interior de sus clases. Zambrano, M. (2007), El hombre y lo divino. México: FCE, p. 86.

22 El único texto que se conserva de este trabajo doctoral que emprende Zambrano junto a Ortega, fue publicado en el tercer número de los Cuadernos de la Facultad de Filosofía y Letras en Madrid, (febrero-marzo) del año 1936.

${ }^{23}$ María acepta entusiasmada la propuesta. Hasta el final de curso y durante todo el año siguiente, Xavier Zubiri irá a su casa diariamente para ser profesor particular, aunque en otras ocasiones será ella quien vaya a casa de Zubiri a recibir clases. Siempre recordará el cuidado con que Xavier la fue llevando por los recovecos de la historia del pensamiento occidental. Durante los primeros meses, leen y comentan la Ética de Spinoza y la Sexta Enéada de Plotino. Corominas, J. \& Vicens, J.A. (2006), Xavier Zubiri. La soledad sonora, Madrid: Taurus, 2006, p. 189. En este punto existe discrepancias entre los estudiosos de Zambrano y Zubiri, debido a que Jesús Moreno en su "Síntesis bibliográfica» sobre María Zambrano, sostiene que aquello que ambos autores leen es la Enéada Tercera de Plotino. Esta referencia sobre la lectura de la Enéada Tercera de Plotino también se encuentra consignado por CASTILlo, J. (1987), «Cronología de María Zambrano», Revista Anthropos, 70-71, p. 75 y en el texto de: A. Savignano, A (2005), María Zambrano: La razón poética. Granada: Comares, p. 33. 
En este punto es necesario recurrir a los actuales antecedentes acerca de la biografía de Xavier Zubiri, que permiten comprender la conexión entre ambos pensadores, además de advertir el significado de las lecturas que ambos realizaban durante esta época, lo que no sólo se explica a partir de la crisis que vivía Zambrano, en relación con su interés por la filosofía, sino también a partir de la crisis que le había generado a Zubiri su ordenación como sacerdote el año 1921. «Zubiri es muy claro cuando señala tajantemente que al ser ordenado sacerdote el 21 de septiembre de 1921 sentía el peso de la nada por su vida; ésta se vaciaba» (Espinoza \& Ascorra, 2012, p. 19). A lo que agrega en una carta dirigida al Obispo de Madrid L. Eijo y Garay el 19 de marzo de 1922: «Mis ideas cada vez más inclinadas a lo que pudiéramos llamar el borde de lo admisible, colocan mi espíritu en crisis continua», Carta de Xavier Zubiri (Corominas \&Vincens, 2006, p. 713).

Este doble vínculo entre Zambrano y Zubiri se expresa en la unión de ambos a partir del trabajo en las aulas de la universidad, que de acuerdo a los lineamientos de Ortega intenta abordar desde lo filosófico la crisis de toda una época, y, en un segundo momento a partir de una unión extrauniversitaria de orden vital, en la cual ambos pensadores enfrentan una crisis de orden personal, estableciéndose de este modo un primer elemento de análisis para la revisión de sus pensamientos. En el caso de Zambrano y Zubiri esta crisis los conduce a efectuar una apuesta por un modo de hacer filosofía, que cuestione radicalmente todo fundamento.

Desde el horizonte universitario, la unión entre ambos se realiza a partir de la formalidad de las aulas Zambrano expresa en su autobiografía que durante toda esta primera etapa como catedrático de la Universidad Central de Madrid, Xavier Zubiri impartirá cursos sobre filosofía griega, haciendo un especial énfasis en la obra aristotélica, actitud que se acrecentará cuando conoce a Heidegger. En su primer libro, Naturaleza, Historia, Dios ${ }^{24}$, recoge parte de lo realizado en sus cursos a partir del año 1926, donde es posible encontrar varios textos dedicados a la filosofía griega y en especial a la obra de Aristóteles. Un punto relevante a destacar de esta primera obra, es que su redacción es posterior tanto al período en que el filósofo trabaja en conjunto con María Zambrano, como al trabajo que realiza con Heidegger, por lo cual en esta obra es posible encontrar elementos de la ontología heideggeriana, que no se encontraban aún en el pensamiento zubiriano, durante su relación con Zambrano. Este hecho queda expresado en la redacción de Naturaleza, historia y Dios, que si bien en éste se da cuenta del trabajo del filósofo, durante el período de cercanía con Zambrano,

24 El libro Naturaleza, historia y Dios fue publicado en el año 1944, pero el trabajo que allí se desarrolla, como el mismo Zubiri lo señala en el prólogo del libro, corresponde principalmente a lo trabajado en sus cursos. «Representan, además, estos escritos la línea general y el espíritu en que he desarrollado mis cursos universitarios desde el año 1926. No puedo menos de pensar con cariño en los discípulos y alumnos de estos años, a quienes he consagrado la mayor parte de mi modesta y silenciosa labor». ZuBIRI, X. (2004), Naturaleza, historia y Dios. Madrid: Alianza, p. 20. 
su redacción posterior conlleva una relectura de su pensamiento desde el horizonte heideggeriano. «En 1935 Zubiri publica en Revista de Occidente, $\mathrm{N}^{\circ} 149$, el ya célebre texto «En torno al problema de Dios» (...) El texto ampliado es el que aparece luego en 1944 en Naturaleza, Historia, Dios (NHD) y es el texto que se ha convertido en todo un clásico. El texto en NHD se amplía, en especial, en el parágrafo II donde se puede ver claramente la raíz heideggeriana de la religación como también su diferencia (Espinoza \& Ascorra, 2012, p. 28).

El vínculo directo entre Xavier Zubiri y Martín Heidegger se inicia a partir de 1928, año en que el filósofo español inicia una estancia de cuatro meses en la aulas de Freiburg, período en el que se enfrenta por primera vez a la figura del filósofo alemán, al que había conocido a partir de la lectura que realizaba Ortega de Ser y tiempo en la Escuela de Madrid, y que conducirá a Zubiri a Freiburg $^{25}$. Esta primera aproximación al filósofo alemán le permitirá a Zubiri, iniciar su propio pensamiento, al distanciarse al final de este proceso de trabajo de heideggeriano, debido a que el filósofo español considera que la filosofía de Heidegger al ser abordada a partir del ser, le faltaba fundamento, el que Zubiri establecerá, posteriormente, a partir de una reflexión acerca de la realidad, en la cual el «ser» sólo corresponde a un momento de la realidad. «Heidegger entiende la realidad como un modo de ser de segundo nivel. En verdad, ve la realidad como el carácter de sustancia y de res extensa de la filosofía tradicional (realismo e idealismo). Sin embargo, como tiene muy claro Zubiri esto es inadmisible en sus supuestos mismos: la realidad no es nada residual de segundo orden» (Espinoza, 2004, p. 221). Zubiri, expresará con los años en el texto Sobre el hombre que: "Dónde la filosofía de Heidegger ha jugado con los conceptos de ser y ente ha fallado, porque hay tres términos: ser, ente y realidad. Con lo cual ha dejado el problema radical de lo que es la realidad» (Zubiri, 1986, p. 676).

Si retomamos el trabajo realizado por ambos pensadores españoles, antes de este significativo viaje que realiza a Alemania Xavier Zubiri, es posible mostrar desde la obra de Zambrano, que gran parte del trabajo realizado en conjunto en las aulas de la universidad, se encuentra cifrado en el estudio de la filosofía griega ${ }^{26}$, «en especial en lo concerniente a las categorías aristotélicas,

25 Para entender la relación Heidegger y Zubiri, véase EsPINOzA, R. (2004), «¿Por qué Herr Kollege no ha hablado usted antes». La crítica de Zubiri a "Sein und Zeit», Cuadernos Salmantinos de Filosofía, XXXI, pp. 201-249. Para un estudio detallado de la relación esencial entre Zubiri y Heidegger se pueden leer los siguientes libros: Nicolás, J. \& EsPinozA, R (eds.) (2006), Zubiri frente a Heidegger. Barcelona: Herder. Este libro está compuesto por un conjunto de artículos especializados de la relación de Zubiri con Heidegger; tales artículos están escritos por los más destacados estudiosos de Zubiri (Gracia, Rivera, González, Conill, Espinoza, etc.). Y véase el Libro: Espinoza, R. (2013), Realidad y ser en Zubiri. Granada: Comares, 2013. Este libro es un estudio detallado de la cuestión del ser a través de un diálogo en lo filosófico entre ambos pensadores.

26 María Zambrano en el texto «La condenación aristotélicas de los pitagóricos» perteneciente al libro El hombre y lo divino, incluye tres notas explicativas sobre el trabajo realizado por Xavier Zubiri en relación con la filosofía griega, que ella declara haber escuchado 
pues no podemos olvidar que Zubiri sugiere a Zambrano, como proyecto de tesis, un estudio que incluye una investigación sobre la obra de Aristóteles ${ }^{27}$ » (Soto García, 2011, p. 236).

Durante el año 1927, María Zambrano también da cuenta de haber asistido a un seminario monográfico acerca de René Descartes dictado por Zubiri, y es precisamente este autor y el tratamiento que realizan ambos de éste, lo que permitirá comprender la novedad que estos autores promueven al dar un giro desde el «yo» a la "persona» en sus obras. Esta discusión, de fines de los años 20 , se comienza a fraguar entre ambos a partir de la crítica del «yo» cartesiano, para en un segundo momento cuestionar incluso el «yo» trascendental que propone la filosofía husserliana, presentando un radical cuestionamiento a partir de estos tópicos de toda la modernidad (esta postura Zubiri la mantendrá hasta el final de su vida) ${ }^{28}$.

Xavier Zubiri el año 1937 dicta en la Universidad de la Sorbona en París, la conferencia Res Cogitans, durante la celebración del tercer centenario del Discurso del Método de Descartes. En esta oportunidad establece las condiciones de su crítica al pensamiento cartesiano, al oponer al carácter dualista que Descartes atribuye al ser humano, "Zubiri en plena Sorbona nos habla de unidad del hombre y de la importancia del cuerpo desde el horizonte del medieval Eriúgena

durante el tiempo que cursa sus estudios universitarios en la ciudad de Madrid. Entre estas notas destaca una sobre la Metafísica de Aristóteles y otra sobre la contraposición, en el modo de abordar el quehacer filosófico, entre Sócrates y Aristóteles. ZamBRANO, M. (2007), El hombre y lo divino. México: FCE, p. 100. La relación de ambos pensadores con la obra de Aristóteles que aquí ha sido señalada fue presentada en el Coloquio Internacional Xavier Zubiri, que se realizó en la ciudad de Valparaíso en el año 2010, y que se encuentra editada por la Editorial Comares de Granada, en un volumen dedicado a la obra de Xavier Zubiri y que reúne varios de los escritos presentados durante aquel congreso. Sin embargo, en esta oportunidad lo que se busca presentar es a través de la revisión de pasajes e itinerarios de vida de ambos pensadores que aquello que se fragua en la Escuela de Madrid es una radical reforma al modo como se concebía el entendimiento y con ello el inicio de la originalidad del pensamiento filosófico de ambos pensadores.

27 Este dato es bastante relevante para comprender la filosofía zambraniana, debido a que en uno de sus libros capitales, El hombre y lo divino, dedica un capítulo completo a la reflexión que se desprende de la filosofía de Aristóteles, «La condenación aristotélica de los pitagóricos», donde expone cuáles son las diferencias conceptuales entre su pensamiento y el realismo filosófico.

28 En el texto, Inteligencia y logos, de su trilogía Inteligencia sentiente, elabora de forma detallada un crítica radical a la intuición del yo que se fragua desde el pensamiento cartesiano y cuyo máximo exponente, desde la elaboración de un yo trascendental, es Husserl. En el $\$ 3$ «Falsas ideas acerca de la evidencia», perteneciente al capítulo VI, «La determinación del Logos en sí misma», expresa con detalle: «Por esto, cuando nos dice Husserl que el principio es la reducción de toda nóesis intencional por lo intuido, a mi modo de ver enuncia algo absolutamente inexacto. Al igual que Descartes, Husserl ha emprendido el camino desde la claridad hacia la cosa; cuando lo que hay que emprender es el camino desde la cosa hacia su claridad. El principio de todos los principios no es la impleción intuitiva, sino algo más radical: la exigencia real de impleción». ZuBIRI, X. (1982), Inteligencia y logos. Madrid: Alianza, pp. 237-238. 
y el moderno Spinoza» (Ascorra \& Espinoza, 2011, p. 1063), cuestionando con ellos la condición secundaría de la res extensa en el pensamiento cartesiano.

«El "cuerpo" del hombre no es un cuerpo como lo es un cuerpo físico. En su realidad concreta es, ante todo, la encarnación de una persona. Desde este punto de vista, la estructura del hombre en tanto que anthropos es bien compleja. El cuerpo es aquello que facilita y dificulta y, en todo caso, conforma y modifica la actualización de las posibilidades de una persona. Es "mio", es mi cuerpo en un sentido bien diferente de los otros cuerpos. Es mío en el sentido en que (en su concreción última) yo soy el cuerpo. Se podría tal vez decir: yo soy un cuerpo personal, yo soy una persona corporal. La naturaleza humana, en lo que tiene de "físico" (en el sentido etimológico de la palabra) es una natura natura. Ella es la decantación de mis posibilidades de existir en el mundo de los cuerpos» (Zubiri, 2002, pp. 297-298).

Esta reflexión acerca del cuerpo permite comprender los saltos especulativos que va realizando Xavier Zubiri, desde un primer momento fenomenológico hasta un último momento noológico de su obra, lo cual se verá reflejado en la reflexión acerca de lo humano que realiza, la cual se encuentra organizada desde el abandono de todo carácter sustancialista para comprenderlo, lo que además de presentarse como una crítica directa al hylemorfismo aristotélicotomista como explicación del hombre, permite acercase al carácter personal de lo humano, en el cual la condición social e histórica de éste adquieren relevancia. «Y para Zubiri es fundamental entender al hombre como persona, por la plasticidad del término y lo usará en toda su obra final, desde la década del 70 hasta su trilogía noológica, pero recordemos que usaba el término ya en su ponencia en París de 1937, cuando nos hablaba de un "corps personnel” (Ascorra \& Espinoza, 2011, pp. 1071-1072)

\section{LA REFORMA DEL ENTENDIMIENTO: DE LA CRISIS DEL «YO» A LA PERSONA HUMANA}

Uno de los primeros elementos que es examinado durante estos años en la Escuela de Madrid es la búsqueda de una reforma del entendimiento que permitiera dar una salida a los problemas de su tiempo, que caracterizada desde las formas de Ortega, se presentará desde una complexión de la razón que transita desde una razón vital a una razón histórica. «En los momentos críticos de la historia se ha hablado siempre de una «Reforma del Entendimiento», de una crítica que el intelecto se hace a sí mismo, volviéndose sobre sí, para tomar conciencia de sus propias fuerzas y, más aún, de sus deficiencias» (Zambrano, 1998, p. 133). A través de este texto de 1937, María Zambrano, da cuenta de la motivación presente en las aulas de la Universidad Central, orientada precisamente a la discusión acerca de una reforma del entendimiento que permitiera superar la crisis presente en la sociedad española, de la cual a la fecha había dado cuenta tanto la generación del 98 como Ortega. «Desde el punto de vista de Abellán — a favor de la aceptación de la tesis sobre la existencia de las historias 
«nacionales» de la filosofía - se testimonia el hecho indiscutible de que la existencia de la cultura nacional de un pueblo no puede dejar de reflejarse en sus actividades filosóficas» ${ }^{29}$ (Jakovleva, 1996, p. 306).

Esto permite pensar que la crisis de España — descrita en el primer y segundo apartado de este artículo- conduce a que la preocupación filosófica de este grupo de pensadores, que conforman la Escuela de Madrid, se encuentre orientada a la búsqueda de una reforma del entendimiento que les permita superar la crisis originada a partir de la reflexión acerca del logos. Y que en el caso particular de Zubiri y Zambrano, se agudizaba a partir de crisis personales, que los impulsaban a la búsqueda de nuevos referentes para el pensamiento ${ }^{30}$.

«Hay dos maneras, por lo pronto, de intentar esta crítica del entendimiento: una, volverse hacia el instrumento mismo, analizar su estructura, su funcionamiento "puro», es decir, con independencia del objeto de su aplicación. Es lo que ha hecho la crítica del idealismo moderno contra el realismo grecomedieval. El otro camino ha sido muchas veces intentado y tal vez, por su misma complejidad, no ha sido logrado de modo tan ejemplar y clásico. Pero quizá exista otra razón para que así nos parezca, y es que esta segunda crítica tenga que realizarse en cada paso fundamental que el hombre dé para orientarse en la profunda realidad que le rodea, es decir, que constituya una tarea ineludible de cada época, de cada salto en la historia, ya que, al parecer, la historia humana sí procedería por saltos. Este segundo modo de hacer crítica del entendimiento incluiría, para ser completa, una conciencia de todo aquello que no entra bajo la luz del entendimiento, o al menos en su existencia. Es cuestión de la razón y de lo irracional que se cruza con la del ser y el no ser» ${ }^{31}$ (Zambrano, 1998, pp. 133-134)

29 «Está totalmente reconocido, —dice él一, que el utilitarismo inglés y su versión pragmática de tipo norteamericana, dan un carácter analítico y sensualista a la filosofía anglo-sajona, profundamente preocupada por el problema del conocimiento; en la filosofía francesa, por el contrario predomina el «esprit géometrique», que tiñe su pensamiento de un racionalismo atento ante todo, las cuestiones de método y a la elaboración de ideas claras y distintas: en la filosofía alemana la característica voluntad de poder del pueblo alemán se traduce en la aspiración hacia grandes sistemas filosóficos de trabada arquitectura, con claro énfasis en la sistematización y una evidente idealidad por el "idealismo" ». AbelLán, J. L. (1979), Historia crítica del pensamiento español. Madrid: Espasa Calpe, p. 33.

30 «Zubiri estaba en crisis permanente de fe; pero su crisis no es solamente una crisis personal, esta es el reflejo individual, social e histórico de una Crisis, de una crisis que toca a toda Europa ya en 1922; crisis de posguerra, de pos-siglo, de pos-positivismo, esto es, de pos-historicismo y psicologismo desde la mano del método científico. Una crisis que toca muy hondo al ser humano, así como lo tocaba a él mismo, pues lo pone en cuestión en lo más radical de su realidad». EsPinoza Lolas, R. \& Ascorra Costa, P. (2012), «Heidegger y Zubiri...y el "el problema de Dios"» Revista Veritas, 27, p. 21.

31 El subrayado es nuestro, y con ello se quiere resalta el intento que se desarrollaba en la Escuela de Filosofía de la Universidad Central de romper con todos los límites establecidos por la filosofía a la razón. Ortega tratará el problema a partir de una «razón vital», que con el paso de los años se transformará en «razón histórica». María Zambrano denominará a esta reforma del entendimiento como «razón combativa», «razón misericordiosa», etc hasta desarrollar en el año 1944 la «razón poética». Sin embargo es Xavier Zubiri quien al final de 
Está búsqueda que se refleja en la obra escrita durante esta época por María Zambrano, también se encuentra presente en la obra de Xavier Zubiri, quien declara en el prólogo a la traducción inglesa de Naturaleza Historia, Dios, que este período de su pensamiento se encontraba caracterizado por la búsqueda de una «Lógica de la realidad» ${ }^{32}$.

Como se señalará, posteriormente, esta preocupación por lo real que se desarrolla en la obra de Zubiri, culminará en una inteligencia sentiente que se relaciona con lo real ${ }^{33}$. Sin embargo, la preocupación de este artículo radica en situar a ambos pensadores dentro del registro de una Escuela, la que durante esta época buscaba hacer un giro en la filosofía, desde las implicancias que conlleva una reforma del entendimiento, que en el caso de Zubiri se comenzaba a articular a partir del pensamiento fenomenológico. José Gaos recuerda aquellos primero años de Zubiri en la Facultad de Filosofía y Letras de la Universidad Central como dedicados a la fenomenología ${ }^{34}$, y para ello cuenta una anécdota que relata el día en que ambos se conocieron:

«De pronto corrió por la Facultad el anuncio de un conferencia de Ortega sobre Don Juan [...] Los compañeros propusimos irnos todos juntos [...] Allí llegó Zubiri [...] del camino me ha quedado una imagen desde hace ya no se cuanto tiempo [...] Es que Zubiri fue explicándome a lo largo del camino la fenomenología entera, volviendo una y otra vez sobre el ejemplo de la rosa: la esencia de la rosa, el noema de la percepción de la rosa, la noesis perceptiva de la rosa...»(Gaos, 2008, pp. 13-14).

su obra extremará del modo más radical esta búsqueda al realizar una reforma no a la razón, sino a la facultad intelectiva, dando cuenta de una «inteligencia sentiente».

32 «En esta etapa de mi reflexión filosófica la concreta inspiración común fue ontología o metafísica. Con ello la fenomenología quedó relegada a ser una inspiración pretérita [...] Era ya una superación incoativa de la fenomenología. Por esto, según me expresaba en el estudio "Qué es saber», lo que yo afanosamente buscaba es lo que entonces llame lógica de la realidad». Zubiri, X. (2004), Naturaleza, Historia, Dios. Madrid: Alianza, p. 14.

33 «La inteligencia sentiente no es potencia, sino facultad; una facultad «una», pero metafísicamente compuesta de dos potencias; la potencia de sentir y la potencia de inteligir. Solamente siendo sentiente es como la inteligencia está facultada para producir intelección. Hay que establecer, pues, una diferencia metafísica entre poder como potencia y poder como facultad». ZuBiri, X. (2006), Tres dimensiones del ser humano: individual, social, histórica. Madrid: Alianza, p. 146.

34 El filósofo español estaba totalmente sumergido en el ámbito filosófico alemán que estaba instaurándose como un nuevo horizonte de pensamiento; comprendía muy bien la fenomenología inaugurada por Husserl y no podemos olvidar que sus dos primeros trabajos propiamente filosóficos de alto nivel están incardinados en la fenomenología. Para aprobar en 1921 la Licenciatura en Filosofía Superior en la Universidad Católica de Lovaina presentó la tesina: Le problème de l'objetive d'aprè Ed. Husserl: La Logique pure. Luego se doctoró ese mismo año en Madrid con la tesis: Ensayo de una teoría fenomenológica del juicio. Esta tesis fue publicada en 1923 y es el primer libro sobre fenomenología de Husserl fuera de Alemania. Ambos textos se pueden encontrar en el volumen Primeros escritos (1921-1926). EsPinoza LoLAS, R. (2004), «¿Por qué, Herr Kollege, no ha hablado usted antes?», Cuadernos Salmantinos de Filosofía, XXXI, p. 204. 
Esta preocupación por el pensamiento husserliano, que desarrolló Zubiri durante la primera época de su trabajo filosófico, le permitirá ir fraguando una contundente crítica al yo cartesiano, crítica que lo que a largo de los años se hará más explícita, en especial en los textos de mediados de los años 60, debido a que a partir de esa época destierra completamente la idea de «sustancia» de su filosofía y con ello culmina la crítica a toda una estructura de pensamiento que otorga a la razón y/o a la conciencia características trascendentes y distintas de aquello que se conoce. «La fenomenología es la gran sustantivación de la conciencia que ha ocurrido en la filosofía moderna desde Descartes. Sin embargo, no hay conciencia; hay actos conscientes» (Zubiri, 2004, p. 15).

$\mathrm{Al}$ revisar la obra de Zubiri es fácil constatar que la crítica al pensamiento moderno se origina a partir de la revisión temprana que realiza el autor de la obra de René Descartes. Joan Albert Vicens, de la Universidad de Barcelona, en el artículo «Xavier Zubiri, Descartes y el origen de la filosofía moderna» efectúa una descripción exhaustiva de las diversas referencias que presenta Xavier Zubiri del pensamiento cartesiano.

«Le dedicó monográficamente el trabajo Res Cogitans, presentado en París en el Congreso Internacional de Filosofía en 1937, la Introducción a la edición en castellano de la correspondencia de Elisabeth de Bohemia y Cristina de Suecia, publicada en 1944 y reeditada en Naturaleza, Historia, Dios, bajo el título de Descartes, también en 1944. Descartes ocupó asimismo un lugar importante en sus cursos universitarios publicados hasta ahora, hay que mencionar "El ser como pensado: de Descartes a Husserl" (1931-32); de lo segundos destaca el capítulo que le dedica en Los problemas fundamentales de la metafísica occidental (1969)» (Vicens, 2011, 67).

Esta preocupación por Descartes no sólo responde a una actitud de revisión monográfica acerca de su obra, sino que también se encuentra ordenada en diversas preocupaciones que la filosofía de Descartes ha instaurado en la historia de la filosofía, y que se encuentra articulada a partir del influjo del pensamiento heideggeriano en Zubiri, pues el pensador alemán, en su obra Ser y tiempo establece condiciones que permiten desautorizar el pensamiento cartesiano como explicación de la mundaneidad y con ello articular toda una crítica a la posición cartesiana ante el conocimiento y el ser humano.

«Descartes agudizó el estrechamiento de la pregunta por el mundo a la pregunta por la coseidad natural considerada como el ente primeramente accesible dentro del mundo. Consolidó la opinión de que el conocimiento óntico presuntamente más riguroso de un ente sería también la vía de acceso al ser primario del ente descubierto en ese conocimiento. Pero es necesario reconocer al mismo tiempo que también los intentos de "completar" la ontología de la cosa se mueven fundamentalmente en la misma base dogmática que Descartes» (Heidegger, 1997, p. 125).

Esta cuestionamiento a Descartes le permite a Xavier Zubiri, de acuerdo a lo que declara Vicens en el texto citado, establecer cuatro preocupaciones principales a partir de la obra del francés: «1) la radicalización del horizonte de la 
nihilidad como supuesto básico de la nueva metafísica, 2) la asimilación de la filosofía a la ciencia, 3) la centralidad del Yo, expresada en el cogito, y finalmente, 4) la reformulación de la noción de verdad y la interpretación de la realidad desde la objetividad» (Vincens, 2011, 68)

Propiamente es la tercera de estas preocupaciones, la que permite adentrarse en la crítica que Zubiri realiza al «yo», y a partir de este ejercicio evidenciar la originalidad de la propuesta que realizará con los años.

«En sus escritos, Descartes ha resbalado sobre el ser del hombre para atenerse solamente a sus operaciones: pensar y querer. Heredero una vez más de la metafísica de su tiempo, acusa Descartes, por un lado, la equivocidad radical con que separa metafísicamente los tres ámbitos de la realidad (Dios, el mundo y el alma), y, de otro, la unívoca indiferenciación conceptual con que entiende el vocablo ser o res como él dice. Y en este juego entre la univocidad conceptual y la equivocidad real, se expresa justamente el dislocamiento entre inteligencia y voluntad, por un lado, el dislocamiento entre alma y realidad cósmica por otro» (Zubiri, 2004, pp. 168-169).

El mismo año que Zubiri participa en el coloquio dedicado a los trecientos años de la publicación del Discurso del método de Descartes, María Zambrano publica en la Hora de España, el texto "La reforma del entendimiento» ${ }^{35}$, en este artículo da cuenta de las críticas que el propio intelecto se hace a sí mismo. Y es en este registro que establece una revisión de cómo ha operado esta crítica a través de la historia de la filosofía, estableciendo a partir de ésta una revisión de la obra cartesiana.

«En el Renacimiento, el filósofo Descartes rompe audazmente con estas ideas y hace tabla rasa de todo lo aprendido para encontrar directamente, de modo inmediato, transparente, una verdad original, hallada por el mismo en respuesta de su propio problema. La encuentra en la existencia de la conciencia. El ser ya no es primaria y radicalmente la realidad física, sino la realidad pensante. El centro de gravitación del pensamiento es ya el ser dado en la conciencia humana. El hombre sabe que existe porque lo encuentra en su pensamiento, que es identificado en la conciencia» (Zambrano, 1998 p. 135).

A partir de estos fragmentos de sus obras es posible evidenciar cómo ambos pensadores, si bien ya no trabajan juntos, y desde hacía años que no se veían o visitaban, presentan como inquietud gravitante dentro de su obra la necesidad de presentar una nueva forma de comprender al ser humano, desde un horizonte diverso al del yo cartesiano, debido a que este último encierra al hombre dentro del solipsismo de su pensamiento.

Diego Gracia, quien ha dedicado gran parte de su trabajo intelectual a la difusión y estudio de la obra de Xavier Zubiri, señala que la preocupación del

35 Este artículo fue publicado en Hora de España en diciembre del año 1937. Actualmente es parte de los artículos de Zambrano agrupados bajo el nombre de Escritos de la guerra civil, los que han sido reeditados por Trotta junto al libro Los intelectuales en el drama de España, obra que la autora redacta y publica en Chile en el año 1937. 
autor por la fenomenología surge como un intento de superación y culminación de la modernidad desde la cual inicia su reflexión filosófica. «Era preciso comenzar desde el principio, rehacer el edificio de la filosofía desde sus mismos cimientos. Eso es lo que se propuso, en concreto, el movimiento conocido con el nombre de Fenomenología» (Gracia, 2012, p. 255). Sin embargo, este intento de superación y culminación de la modernidad, que inicia Zubiri desde la fenomenología, permite considerar que aquello que realiza el autor, no sólo es una crítica a Descartes, sino también al modo como este paradigma para comprender al ser humano conlleva a que precisamente éste se desvincule de lo real.

«Zubiri a lo largo de los años cambió drásticamente su concepción sobre la estructura precisa de las notas constitutivas de la realidad humana. En el famoso curso Cuerpo y alma (1950-1951) y sobre todo, en el curso El problema del hombre (1953-1954) había dado al alma una sustantividad e independencia, que más tarde le parecieron excesivas. Su explicación de la unidad del hombre, a pesar de intentar superar los dualismos, seguía siendo hilemórfica y trataba de asegurar algunas de las explicaciones tradicionales que atribuían al alma una inmortalidad para su propia naturaleza. Todavía en «El hombre realidad personal»(1964), a pesar de los esfuerzos conceptuales y terminológicos, no se libera de lo que pudiéramos llamar presiones dogmáticas. Sólo más tarde y expresamente en «El hombre y su cuerpo» (1973) empieza a llevar a sus últimas consecuencias su idea de la unidad estructural entre lo psíquico y lo orgánico. Sin negar nunca la irreductibilidad de lo psíquico humano a lo orgánico, cada vez mantuvo más firmemente su rigurosa unidad y codeterminación mutua, de modo que no puede darse uno sin el otro (...) Zubiri acabó pensando y afirmando que la psique es por naturaleza mortal y no inmortal, de modo que con la muerte acaba todo en el hombre o acaba el hombre del todo» (Ellacuría, 1986, pp. XVII-XVIII).

Este texto de Ignacio Ellacuría no tiene como objetivo presentar el decurso de la obra de Zubiri desde el yo, a la construcción o elaboración de la noción de persona en su pensamiento, sino presentar la crítica de la época, que conduce a los pensadores de la Escuela de Madrid, hacia la búsqueda de nuevos referentes para el pensamiento, y a partir de ello para el ser humano. Por esta razón, en este punto es necesario retomar los elementos que determinaban la vida del autor por aquella época, pues a partir de ellos es posible comenzar a articular el decurso de su pensamiento. Antonio Pintor-Ramos en el texto «Zubiri y la historia de la filosofía» señala:

«En primer lugar, Zubiri había obtenido en 1926 la cátedra de Historia de la Filosofía y Letras de la universidad de Madrid y se vio forzado por su trabajo profesional a enfrentarse de modo directo con el conjunto de la filosofía occidental para explorar allí sus posibilidades como camino para el ejercicio la actividad filosófica. En segundo lugar, poco después estudia Ser y tiempo de Heidegger y en 1929-30 sigue alguno de sus cursos en Friburgo de Brisgovia; de la profunda huella que en este momento le deja el contacto con Heidegger saca Zubiri dos conclusiones importantes para nuestro tema: la importancia de la historicidad y el relieve peculiar y único que dentro de la historia de la filosofía corresponde a los griegos» (Pintor-Ramos, 2012, pp. 14-15). 
Esta primera conclusión acerca de la historicidad — que destaca Pintor Ramos- es la que le permitirá a Zubiri plantearse al ser humano, desde una perspectiva distinta a la que hasta el momento se había abordado a lo largo de la historia de la filosofía (dualismo, hylemorfismo, racionalismo, etc), transformándose en uno de los elementos que permitirá con el tiempo fisurar todo registro sustancialista atribuido a lo humano ${ }^{36}$.

Entonces, la facultad del entendimiento que se pone en crisis, a partir de la revisión de los tópicos cartesianos, es la que le permite a Zubiri con los años ir dirigiendo su atención hacia la persona, entendiendo por ello a un ser humano que no posee un carácter clausurado sobre sí mismo, sino una vinculación con la alteridad que determina y condiciona la vida humana.

«Las otras personas no son sólo algo con lo que hago mi vida, son también algo que de algún modo soy yo mismo, este hecho tiene un fundamento filético y otro personal. En la realidad del dinamismo de la convivencia tienen su base los dos modos esenciales de la vida colectiva del hombre, el social y el histórico. El hombre vive socialmente según lo que colectivamente se está queriendo, pensando, sintiendo y haciendo, y vive históricamente según lo que colectivamente se quiso, se pensó, se sintió y se hizo en el pasado y lo que en el futuro se quiere y se puede hacer» (Melero Martínez, 2001, p. 77).

En el caso de Zambrano está búsqueda partirá desde una reflexión acerca del alma, la que inicia en 1934 año en que publica el artículo Hacia un saber sobre el alma, uno de los textos con los que comienza a fraguarse su pensamiento. Cuando Ortega conoce el texto cita a Zambrano a su despacho para reprenderla, señalándola que ella recién ha comenzado su formación, por lo tanto no puede pretender que tan tempranamente pueda elaborar una filosofía propia.

36 En el artículo «El hombre y su cuerpo en Xavier Zubiri», José María Melero Martínez, realiza una revisión de los textos antropológico de la obra del autor, lo que permite periodizar y secuenciar el pensamiento del autor desde la crítica del yo cartesiano a la persona. «Puestos en orden cronológico y tomando el tema con la suficiente amplitud nos encontramos con la siguiente secuencia de escritos [Escritos antropológicos de Zubiri]: El problema de Dios (1935); El acontecer humano. Grecia y la pervivencia del pasado filosófico (1942); Tres definiciones clásicas del hombre (curso oral de 33 lecciones) (1946-1947); Cuerpo y alma (Curso oral de 34 lecciones) (1950-1951); La libertad (Curso oral de 33 lecciones) (1951-1952); El problema del hombre (Curso oral de 35 lecciones) (1953-1954); El problema del hombre (1959); Sobre la persona (Curso oral de 5 lecciones); Sobre la voluntad (Curso oral de 5 lecciones) (1961); El hombre realidad personal (1963); El origen del hombre (1964); El hombre y la verdad (Curso oral de 2 lecciones); Notas sobre la inteligencia humana (1967); El hombre: lo real y lo irreal (Curso oral de 6 lecciones); El hombre y el problema de Dios (Curso oral de 6 lecciones) (1968); Estructura dinámica de la realidad (Curso oral de 14 lecciones) (1969); El problema teologal del hombre (Curso oral de 26 lecciones) (1971-1972); El hombre y Dios (Curso oral de 12 lecciones) (1973); El hombre y su cuerpo (1973); Tres dimensiones del ser humano: individual, social e histórica (Curso oral de 3 lecciones); La dimensión histórica del ser humano (1974); La realidad humana (inédito) (1974); El problema teologal del hombre (1975); La concreción de la persona humana (inédito) (1975); El hombre y Dios (1982-1983); La génesis de la realidad humana (inédito) (1982-1983)». Melero Martínez, J. M. (2001) «El hombre y el cuerpo en Xavier Zubiri», Ensayos. Revista de la Facultad de Educación, 16, p. 70. 
«Publica [1934] los cuatro artículos que significan el arranque de su pensar propio: en la primavera, el desolado y radical «Límite de la nada»; en el verano, el ya tan musical «Por qué se escribe»; en el otoño, el fenomenológico "Ante la introducción a la teoría de la ciencia de Fichte», y en el invierno, el proyecto entero de su filosofía "Hacia un saber el alma», que le costó la reprimenda del maestro, quien la llamó a su despacho, la recibió de pie y le dijo: «No ha llegado Ud. aquí (señalándose en el pecho) y ya se quiere ir lejos». María salió de la entrevista llorando por la Gran Vía» (Moreno Sanz, 2004, p. 47).

La diferencia que se expresa entre el pensamiento de Ortega y Zambrano en este artículo radica en la concepción del alma, pues durante este mismo año en el texto Vitalidad, alma y espíritu, Ortega expone la supremacía del espíritu o razón, sobre la esfera afectiva con la que caracteriza al alma ${ }^{37}$. En cambio, Zambrano tomando estas coordenadas del texto de su maestro publica un artículo que establece la necesidad de buscar un nuevo tipo de saber que se base precisamente en el alma, en oposición a la clausura cartesiana del yo, que se esconde tras el horizonte de espíritu, según declara Ortega cuando establece una topografía de la intimidad humana en el artículo mencionado. En definitiva, aquello que Zambrano muestra en este texto es una discrepancia radical con el pensamiento de su maestro, al instalar en el alma el punto desde donde realizar la reforma del entendimiento que buscaba.

«Era necesario una idea del hombre íntegro y aun idea de la razón integra también. Mientras el hombre fuese ente de razón nada más y esta razón fuese la matemática, por ejemplo, ¿Cómo iba a ser posible este saber acerca del alma? Desde este punto de vista sólo era posible un Tratado del alma, de Spinoza, en que el alma es la idea adecuada del cuerpo y nada más» (Zambrano, 1950, p. 22).

En el decurso de su obra estos planteamientos se irán radicalizando, al punto de señalar que los principios de la modernidad, que se inauguran a partir del yo cartesiano han conducido a la crisis de Occidente. «A principios del siglo XVII con Descartes, ocurre un hecho sumamente grave también, y por lo pronto espléndido, y es la definición de hombre como conciencia. A partir de ahí, la idea de que el hombre pende sólo de sí mismo» (Zambrano, 1950, p. 99).

Este horizonte se verá reflejado en la obra de Zambrano, en el libro Persona y democracia, publicado en Puerto Rico en 1958. María Zambrano en este texto y buscando una reflexión acerca de los efectos en el ser humano de esta crisis de Occidente considera que pueden ser evidenciados en la figura de Edipo, en quien se concentra la desdicha de lo humano, pues si bien éste responde al enigma de la esfinge, al señalar que el resultado de su acertijo era el hombre,

37 «En efecto: entre la vitalidad, que es, en cierto modo, subconsciente, oscura y latente, que se extiende al fondo de nuestra persona como un paisaje al fondo de un cuadro, y el espíritu, que vive sus actos instantáneos de pensar y querer, hay un ámbito intermedio más claro que la vitalidad, menos iluminado que el espíritu y que tiene un extraño carácter atmosférico. Es la región de los sentimientos y emociones, de los deseos, de los impulsos y apetitos: lo que vamos a llamar, en sentido escrito alma». Ortega y Gasset, J. (1924) «Vitalidad, Alma y Espíritu», en (1983) Obras Completas, II. Madrid: Alianza, p. 462. 
Edipo $^{38}$ no sabía quién era él; esta tragedia evidencia la crisis instaurada en Occidente a partir de la presentación del ser humano reducido a sujeto epistemológico, esto conducirá a que el carácter de persona en la obra de Zambrano, intente romper con toda la herencia cartesiana de pensamiento, y la persona en su obra adquiera una condición de orden político insospechada, porque «somos necesariamente persona».

"Convivir quiere decir sentir y saber que nuestra vida, aun en su trayectoria personal, está abierta a la de los demás, no importan sean nuestros próximos o no; quiere decir saber vivir en un medio donde cada acontecer tiene su repercusión, no por inteligible menos cierta; quiere decir saber que la vida es ella también en todos sus estratos sistema» (Zambrano, 1958, pp. 25-26).

\section{Conclusión}

A partir de los señalado en el texto lo que se quiere resaltar es que la Escuela de Madrid, como movimiento filosófico de renovación del pensamiento español, no sólo debe ser observado desde aquello que Abellán describe como los elementos propios de esta escuela, sino revisar la relación existente entre los pensadores que la componen como un modo de establecer con claridad el impacto y novedad del pensamiento español, que se fragua en la Escuela de Filosofía de la Universidad Central de Madrid, que tal como se ha señalado en el texto se articulaba desde una mirada de crisis.

Esta denominada crisis de Occidente - de acuerdo con Zambrano- se organiza a partir de las coordenadas antropocéntricas de la modernidad que conduce a la reducción del ser humano a un sujeto solipsista, el cual se encuentra completamente desvinculado de su entorno y de los otros.

Zambrano y Zubiri, siguiendo el decurso de estas miradas intentarán con los años a partir del concepto de persona superar esta mirada del sujeto sobre sí mismo, y sobre su conocimiento, para explorar nuevas concepciones antropológicas en las que se destaque al ser humano no desde la clausura en sí mismo sino desde la relación o convivencia con los otros, en el caso de Zubiri esto se verá reflejado en su noología, y en el caso de Zambrano en la presentación de un método de pensamiento que cuestiona la homogeneidad del sujeto moderno a partir de la crítica la linealidad que se le atribuye a la temporalidad.

38 «Hay un protagonista entre todos, un hombre Edipo, en quien se concentra la desdicha de la condición humana; pues es culpable siendo inocente; comete el peor de los delitos habiendo huido de ello, porque no sabe quién es. Si Edipo hubiese podido reconocer a sus padres y a su madre, no hubiese matado aquél y esposado aquella. Habría descifrado el enigma propuesto por la Esfinge. Irónicamente la contestación era: el hombre. Mas después de haber acertado no sabía qué era el hombre, ni quién era él, este hombre, Edipo. El conflicto de ser hombre queda pues planteado no en términos de "existir" o de "ser o no ser", sino en términos de conocimiento». Zambrano, M. (1996), Persona y Democracia. Madrid: Siruela, p. 72. 


\section{Bibliografía}

\section{LIBROS}

Abellán, J. L. (1979), Historia crítica del pensamiento español, Madrid: Espasa Calpe. Corominas, J. \& Vicens, J. A. (2006), Xavier Zubiri. La soledad sonora. Madrid: Taurus. Gaos, J. (2008), Filosofía de la filosofía. México: FCE.

Espinoza, R. (2013), Realidad y ser en Zubiri. Granada: Comares, 2013.

HeIDEgGer, M. (1997), Ser y tiempo. Santiago de Chile: Universitaria.

Marías, J. (1959), La escuela de Madrid. Estudios de filosofía española. Buenos Aires: EMECE.

Modin, I. (1994), Ortega y Gasset y la cultura latinoamericana. México: FCE.

Nicolás, J. \& Espinoza, R (eds.) (2006), Zubiri frente a Heidegger. Barcelona: Herder.

Savignano, A. (2005), María Zambrano: La razón poética. Granada: Comares.

Zambrano, M. (1950), Hacia un saber sobre el alma. Buenos Aires: Losada.

- (1989), Delirio y Destino. Madrid: Mondadori.

- (1996), Persona y Democracia. Madrid: Siruela.

- (1998), Los intelectuales en el drama de España y escritos de la guerra civil. Madrid: Trotta.

- (2002), Cartas a la Pièce, Correspondencia con Agustín Andreu. Valencia: Pre-Textos.

- (2004), Pensamiento y poesía en la vida española. Madrid: Biblioteca Nueva.

- (2007), El hombre y lo divino. México: FCE.

ZUBIRI, X (1982), Inteligencia y logos. Madrid: Alianza.

- (2002), Sobre el problema de la filosofía y otros escritos. Madrid: Alianza.

- (2004), Naturaleza, historia y Dios. Madrid: Alianza.

- (2006), Sobre el hombre. Madrid: Alianza.

- (2006), Tres dimensiones del ser humano: individual, social, histórica. Madrid: Alianza.

\section{CAPÍTULOS DE LIBRO}

Gracia, D. (2011) «La antropología de Zubiri» en Nicolás, J. A. (2011), Guía Comares de Zubiri. Granada: Comares, pp. 251-276.

Ellacuría, I. (1986) «Presentación», Zubiri, X. (1986), Sobre el hombre. Madrid: Alianza, pp. IX-XXIII

Moreno Sanz, J. (2004), "Síntesis biográfica», Moreno, J. y Muñoz, F. (eds.) (2004) en María Zambrano 1904-1991. De la razón cívica a la razón poética. Madrid: Residencia de Estudiantes, pp. 37-80.

- (2004), «Guías y Constelaciones», Moreno, J. y Muñoz, F (eds.) (2004) en María Zambrano 1904-1991. De la razón cívica a la razón poética. Madrid: Residencia de Estudiantes, pp. 209-251.

Ortega y Gasset, J. (1914), «Meditaciones del Quijote» en (1983) Obras Completas, I. Madrid: Alianza, pp. 309-400.

- (1924), «Vitalidad, Alma y Espíritu», (1983) Obras Completas, II. Madrid: Alianza, pp. 451-480.

- (1934), «Prologo para alemanes», en (1962) Obras Completas, VIII. Madrid: Alianza, pp. 11-58.

Pintor Ramos, A. (2011), «Zubiri y la historia de la filosofía», Nicolás, J. A. (2011), Guía Comares de Zubiri. Granada: Comares, pp. 9-25.

Soto García, P. (2011), "Convergencias y divergencias entre Xavier Zubiri y María Zambrano», Nicolás J. A. (eds) (2011), Guía Comares de Zubiri. Granada: Comares, pp. 229-248. 
Vicens, J. A. (2011) «Xavier Zubiri, Descartes y el origen de la filosofía moderna», en. Nicolás, J. A (ed) (2011), Guía Comares de Zubiri. Granada: Comares, pp. 65-95.

\section{REVISTAS}

Ascorra Costa, P. \& Espinoza Lolas, R. (2011), «El cuerpo y el alma en Zubiri. Un problema filosófico-teológico», Pensamiento, 67, pp. 1061-1075.

Camazón Linacero, J. P. (2000), «La crisis europea en Revista de Occidente (1923-1936)», Espacio, Tiempo y Forma, 13, pp. 369-391

CAmpomar, M. (2004) «El perspectivismo americano de Ortega y Gasset», Revista de Occidente, 276, pp. 153-186.

Esmacés Sánchez, J. J. (1993) «Ortega y Gasset (1883-1955)», Perspectivas: Revista trimestral de educación comparada, XXII, París: UNESCO Oficina Internacional de Educación, pp. 808-821.

Espinoza, R. (2004), “¿`Por qué, Herr Kollege, no ha hablado usted antes?”. La crítica de Zubiri a "Sein und Zeit"», Cuadernos Salmantinos de Filosofía, XXXI, pp. 201-249.

Espinoza Lolas, R. \& Ascorra Costa, P. (2012), «Heidegger y Zubiri...y el "el problema de Dios"», Revista Veritas, 27, pp. 9-33.

JAKovLEVA, L. E. (1996), «José Abellán y la "especificidad” de la filosofía española», Anales del Seminario de Historia de la filosofía, 13, pp. 305-318.

Melero Martínez, J. M. (2001) «El hombre y el cuerpo en Xavier Zubiri», Ensayos. Revista de la Facultad de Educación, 16, pp. 69-84.

Zambrano, M. (2004) «La razón que se busca (A propósito de la razón vital)», Revista de Occidente, 276, pp. 89-119.

Pontificia Universidad Católica de Valparaíso PAMELA Soto GARcía y RicARdo EsPINOZA Lolas pamela.soto@ucv.cl

[Artículo aprobado para publicación en este número extraordinario en noviembre de 2014] 
